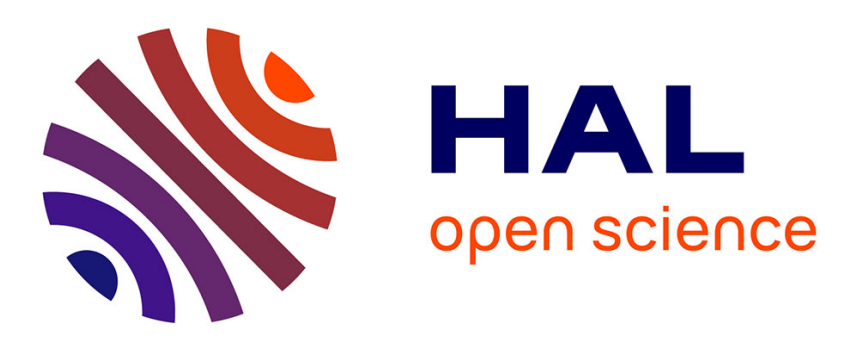

\title{
On Output-based Sliding Mode Control Design Using Minimax Observer
}

Sergiy Zhuk, Andrey Polyakov

\section{To cite this version:}

Sergiy Zhuk, Andrey Polyakov. On Output-based Sliding Mode Control Design Using Minimax Observer. International Workshop on Variable Structure Systems, Jun 2014, Nantes, France. hal$01071233 \mathrm{v} 2$

\section{HAL Id: hal-01071233 \\ https://hal.inria.fr/hal-01071233v2}

Submitted on 7 Oct 2014

HAL is a multi-disciplinary open access archive for the deposit and dissemination of scientific research documents, whether they are published or not. The documents may come from teaching and research institutions in France or abroad, or from public or private research centers.
L'archive ouverte pluridisciplinaire HAL, est destinée au dépôt et à la diffusion de documents scientifiques de niveau recherche, publiés ou non, émanant des établissements d'enseignement et de recherche français ou étrangers, des laboratoires publics ou privés. 


\title{
On Output-based Sliding Mode Control Design Using Minimax Observer
}

\author{
Sergiy Zhuk and Andrey Polyakov
}

\begin{abstract}
The classical problem of the sliding mode control design, which guarantees finite-time reaching and further system motion on a linear hyperplane, is considered for the linear time-invariant disturbed system with the noised measurements of the output. The control law, which provides to the closed-loop system the optimal reaching (as close as possible) of the selected sliding surface, is designed using minimax state observer. The case of discontinuous and continuous admissible feedbacks are studied. The theoretical results are supported by numerical simulations.
\end{abstract}

\section{INTRODUCTION}

Sliding mode is the oldest robust control technique introduced more that 50 years ago (see, for example, [21] and references therein). This method had opened new research areas from purely theoretical domains to practical applications. The main theoretical advantage of sliding mode control is its insensitivity to the so-called matched disturbances and uncertainties, see [7], [22], [20].

The control practice frequently needs designing the sliding modes for systems with mismatched uncertainties [1], [17], [23] as well as for output-based feedback control application [6], [20]. Realization of all classical sliding mode control ideas is complicated for such systems. So, finding the conditions, when the sliding mode control methodology is still useful, is very important.

This paper treats the problem of output-based sliding mode control design for a linear plant with matched additive exogenous disturbances and bounded deterministic measurement noises. In this case, ensuring of the ideal sliding mode in the state space of the original system is impossible due to incomplete and noised measurements. The control law, which provides the motion of the closed-loop system as close as possible to the selected sliding surface, can only be designed. It is worth to stress that just the conventional (first order) sliding mode control design principles are studied in the paper. It is known (see, for example, [2]) that realization of the reaching phase of the sliding surface can be formulated as a special optimization problem. This paper shows that for the case of noised output measurements, the sliding mode control algorithm is just one possible solution of the corresponding optimization problem, which, in fact, admits both continuous and discontinuous optimal control laws.

The present research is partially supported by ANR ChaSliM project Sergiy Zhuk is with IBM Research, Server 3, Damastown Ind. Park, Dublin 15, Ireland, sergiy.zhuk@ie.ibm.com

Andrey Polyakov is with NON-A, INRIA - LNE, Parc Scientifique de la Haute Borne 40, avenue Halley Bat.A, Park Plaza 59650 Villeneuve d'Ascq, andrey.polyakov@inria.fr and with LAGIS UMR CNRS 8219, Ecole Centrale de Lille, BP 48, Cité Scientifique, 59651 Villeneuve-d'Ascq, France.
The design procedure is essentially based on linear minimax estimation technique for linear dynamical systems (see, for instance, [24] and references therein). We refer the reader to [13], [4], [10] for the basic information on the minimax framework. The minimax observer presents the best possible guaranteed estimate of the system state and the sliding variable. This property allows us to reduce the problem of sliding mode control design in the state space of the original system to the sliding mode realization for the observer's variables.

It is worth to stress that the minimax observer studied in the paper is the optimal over all observers, which are represented by linear functionals defined in $\mathbb{L}_{2}$ - spaces. The paper also presents a numerical comparison of the precision of the optimal control laws designed by minimax observer and the fixed-time feedback [15] based on the second order sliding mode (2-sm) observer [5], which is nonlinear. We refer the reader to [20], [7], [22], [18] for more information about sliding mode observers.

The paper is organized as follows. The next section present the notations used in the paper. Then the problem statement and basic assumptions are considered. The minimax observed design is given in the section 4 . Next the control design algorithms are discussed. Finally, the numerical simulations and conclusions are presented.

\section{NOTATION}

Through the paper the following notations will be used:

- $\mathbb{R}_{+}=\{x \in \mathbb{R}: x>0\}, \mathbb{R}_{-}=\{x \in \mathbb{R}: x<0\}$, where $\mathbb{R}$ is the set of real number;

- $\|\cdot\|$ is the Euclidian norm in $\mathbb{R}^{n}$, i.e. $\|x\|=$ $\sqrt{x_{1}^{2}+\ldots+x_{n}^{2}}$ for $x=\left(x_{1}, \ldots, x_{n}\right)^{T} \in \mathbb{R}^{n}$;

- if $P \in \mathbb{R}^{n \times n}$ then the inequality $P>0(P \geq 0$, $P<0, P \leq 0)$ means that $P$ is symmetric and positive definite (positive semidefinite, negative definite, negative semidefinite).

- $\mathbb{L}_{[a, b]}^{2}$ is a set of Lebesgue quadratically integrable functions defined on $[a, b]$.

\section{Problem Statement}

Consider the linear output control system

$$
\begin{gathered}
\dot{x}=A x+b(u+g(t)), \\
y=C x+w(t), \\
t \in[0, T), \quad x(0)=x_{0} \in \mathbb{R}^{n},
\end{gathered}
$$

where

- $T \in R_{+}$is a finite instant of time or $T=+\infty$, 
- $x \in \mathbb{R}^{n}$ is the vector of system state,

- $u \in \mathbb{R}$ is the scalar control input,

- $y \in \mathbb{R}^{k}$ is the measured output,

- the function $g: \mathbb{R}_{+} \rightarrow \mathbb{R}^{p}, p \leq n$ describes the matched external disturbances and

- the function $w: \mathbb{R}_{+} \rightarrow \mathbb{R}^{k}$ is a deterministic measurement noise,

- the system parameters $A \in \mathbb{R}^{n \times n}, b \in \mathbb{R}^{n}, C \in \mathbb{R}^{k \times n}$ are assumed to be known and time-invariant.

We study this system under the standard assumptions ( see,[22], [7]).

Assumption 1: The pair $(A, C)$ is observable, the pair $(A, b)$ is controllable.

The noise measurements $w \in \mathbb{L}_{[0, T]}^{2}$ and exogenous disturbances $g \in \mathbb{L}_{[0, T]}^{2}$ are assumed to be deterministic and satisfy the restriction

$$
x_{0}^{T} P_{0}^{-1} x_{0}+\int_{0}^{T} w^{T}(\tau) R w(\tau)+Q g^{2}(\tau) d \tau \leq 1,
$$

where $P_{0} \in \mathbb{R}^{n \times n}, R \in \mathbb{R}^{k \times k}$ are symmetric positive definite matrices and $Q \in \mathbb{R}_{+}$is a positive number.

The admissible control law is assumed belonging to $\mathbb{L}_{[0, T]^{-}}$ space, which contains both continuous and discontinuous functions.

The classical control problem ( see, [22], [7]) for the system (1) is to design the control algorithm, which realizes finite-time reaching of a given linear plane like

$$
f^{T} x=0, \quad f \in \mathbb{R}^{n}, \quad f^{T} b \neq 0,
$$

and further sliding on this plane. It is worth to stress that the condition $f^{T} b \neq 0$ is necessary for realization of the first order sliding mode control principles.

Let us consider the sliding mode control design problem for the reaching phase, i.e. we need to find the control law $u$ such that $f^{T} x(T)=0$. The considered problem can be equivalently rewritten

$$
\begin{gathered}
\left\|f^{T} x(T)\right\| \rightarrow \min \\
\text { s.t. (1) - (3). }
\end{gathered}
$$

Indeed, obtaining a solution of this optimization problem with zero value of the cost functional guarantees the successful realization of the reaching phase. Due to measurement noises and system disturbances, the sliding mode of the given surface $f^{T} x=0$ may not be guaranteed. In this case it is important to know, which sort of feedback control will be optimal in order to provide the system motion as close as possible to the surface.

In this paper we only study the observer-based feedback design assuming that static output-based sliding mode control (see, for example, [20]) can not be applied, i.e. $f \notin$ range $\left(C^{T}\right)$.

\section{Min-Max Optimal State Observer Design}

According the classical methodology of the sliding mode control design, the precise knowledge of the so-called sliding variable $s(t):=f^{T} x(t)$ is required in order to ensure the motion of the system (1) on the surface $f^{T} x=0$. We stress that this information is not available as the given output $y(t)$ is incomplete and noisy. In this situation, the best available information about the value of $f^{T} x(t)$ is represented by the minimax estimate of the state.

Let $x_{u}, x_{g}$ denote the solutions of the following ODEs:

$$
\begin{array}{ll}
\frac{d x_{u}}{d t}=A x_{u}+b u, & \bar{x}_{u}(0)=0, \\
\frac{d x_{g}}{d t}=A x_{g}+b g, & x_{g}(0)=x_{0} .
\end{array}
$$

Then, clearly, $x(t)=x_{u}(t)+x_{g}(t)$ and

$$
y_{g}(t):=y(t)-C x_{u}(t)=C x_{g}(t)+w(t) .
$$

The function $x_{g}$ may be considered as a noisy part of $x$ corresponding to disturbances from the ellipsoid (3) and $x_{u}$ represents its "mean" value corresponding to the case of zero disturbances $x_{0}=0$ and $g=0$, which forms (together with $w=0$ ) the central point of the ellipsoid (3). Since $x_{g}(t)$ does not depend on the control parameter $u$ we may first construct an estimate of the noisy part. Following [24] we introduce the following definition.

Definition 1: Assume that $l \in \mathbb{R}^{n}$ and $\widehat{U} \in L^{2}\left(0, t^{*}\right)$. A linear functional

$$
\widehat{\mathcal{U}}_{l}(y):=\int_{0}^{t^{*}} \widehat{U}^{T}(\tau) y(\tau) d \tau
$$

is called a minimax estimate of $l^{T} x(t)$ iff

$$
\begin{aligned}
\sigma\left(\widehat{U}, l, t^{*}\right) & :=\sup _{\left(x_{0}, g, w\right) \in \Omega^{*}}\left(l^{T} x\left(t^{*}\right)-\widehat{\mathcal{U}}_{l}(y)\right)^{2} \\
& \leq \sigma\left(U, l, t^{*}\right), \quad \forall U \in L^{2}\left(0, t^{*}\right),
\end{aligned}
$$

where $\Omega^{*}$ is defined by (3) with $T=t^{*}$.

The number $\hat{\sigma}\left(l, t^{*}\right):=\sigma\left(\widehat{U}, l, t^{*}\right)$ is called the minimax estimation error.

In other words, the minimax estimate $\widehat{\mathcal{U}}_{l}$ has minimal estimation error $\hat{\sigma}$.

Proposition 1: Let $\hat{x}_{g}(t) \in \mathbb{R}^{n}$ be the solution of the following ODE:

$$
\left\{\begin{array}{l}
\frac{d \hat{x}_{g}}{d t}=A \hat{x}_{g}+P(t) C^{T} R\left(y_{g}(t)-C \hat{x}_{g}\right), \\
\hat{x}_{g}(0)=0,
\end{array}\right.
$$

where $P(t) \in \mathbb{R}^{n \times n}$ is the solution of the following differential Riccati equation:

$$
\left\{\begin{array}{l}
\dot{P}=A P+P A^{T}+Q^{-1} b b^{T}-P C^{T} R C P, \\
P(0)=P_{0} .
\end{array}\right.
$$

Then $\widehat{\mathcal{U}}_{l}\left(y_{g}\right)=l^{T} \hat{x}_{g}\left(t^{*}\right)$ and $\hat{\sigma}\left(l, t^{*}\right)=\left(l^{T} P\left(t^{*}\right) l\right)^{\frac{1}{2}}$, where $y_{g}$ is defined by (6).

The detailed proof of this proposition is available in the literature (see for instance [10], [24]). We refer the reader to Appendix for the sketch of the proof.

Let us stress that for an observable pair $\{A, C\}$ and a controllable pair $\{A, b\}$ the proposed observer is stable, that is $A-P^{\infty} C^{T} R C$ is a stable matrix provided $P^{\infty}$ solves the algebraic Riccati equation:

$$
0=A P+P A^{T}+Q^{-1} b b^{T}-P C^{T} R C P
$$


The proof of this fact see, for example, in [19].

The definition of the minimax estimate $\widehat{U}$ implies that

$$
\left(l^{T} x_{g}\left(t^{*}\right)-l^{T} \hat{x}_{g}\left(t^{*}\right)\right)^{2} \leq l^{T} P\left(t^{*}\right) l .
$$

Now we recall that $x\left(t^{*}\right)=x_{u}\left(t^{*}\right)+x_{g}\left(t^{*}\right)$ and so

$$
x\left(t^{*}\right)=x_{u}\left(t^{*}\right)+\hat{x}_{g}\left(t^{*}\right)+e\left(t^{*}\right) .
$$

where $e\left(t^{*}\right)=x_{g}\left(t^{*}\right)-\hat{x}_{g}\left(t^{*}\right)$ and $l^{T} e\left(t^{*}\right) \leq\left(l^{T} P\left(t^{*}\right) l\right)^{\frac{1}{2}}$ does not depend on $u$. Define

$$
\hat{x}:=x_{u}+\hat{x} .
$$

Then it is straightforward to check that:

$$
\left\{\begin{array}{c}
\frac{d \hat{x}}{d t}=A \hat{x}+P(t) C^{T} R(y(t)-C \hat{x})+b u(t), \\
\hat{x}(0)=0 .
\end{array}\right.
$$

Since the calculations above hold true for any $0<t<t^{*}$, we obtain:

$$
x(t)=\hat{x}(t)+e(t),
$$

where the estimation error satisfies the inequality

$$
l^{T} e(t) \leq\left(l^{T} P(t) l\right)^{\frac{1}{2}}
$$

and the latter estimate does not depend on the control $u$. The inequality (9) holds for all $l \in \mathbb{R}^{n}$ proving the following optimal (in the minimax sense) guaranteed estimate of the system state

$$
x(t) \in\left\{z \in \mathbb{R}^{n}: \quad z=\hat{x}_{u}(t)+e, \quad e^{T} P^{-1}(t) e \leq 1\right\},
$$

i.e. the state vector $x(t)$ belongs to the ellipsoid centered at $\hat{x}(t)$ with the shape matrix $P(t)$. Recall that the formula (9) with $\forall l \in \mathbb{R}^{n}$ is just a way to define the ellipsoid (see, for example, [10]).

It is worth to stress that the minimax approach to observer design provides the exact estimate of the system state, namely, for any $e_{*} \in \mathbb{R}^{n}$ belonging to the estimating ellipsoid (i.e. $\left.e_{*}^{T} P^{-1}(t) e_{*} \leq 1\right)$ and for any $t \in[0, T]$ there exist $x_{0}^{*} \in \mathbb{R}^{n}, w^{*} \in \mathbb{L}_{2}$ and $g^{*} \in \mathbb{L}_{2}$ satisfying (3) such that the equality $x(t)=\hat{x}_{u}(t)+e^{*}$ holds.

Note that $P$ does not depend on the control parameter explicitly. This suggests to design a controller as a function of the center of the ellipsoid, that is $\hat{x}_{u}$. The next section presents the controller design.

\section{Control Design}

Now let us consider the problem of the reaching phase realization of the sliding mode control for the system (1)(3), which is equivalently rewritten in the form (4).

Denote the sliding variable by

$$
\sigma=f^{T} x .
$$

Using the formula (10) we derive

$$
\sigma(T)=f^{T} x(T)=f^{T} \hat{x}(T)+f^{T} e,
$$

where the state estimate $\hat{x}$ satisfies (8) and $e$ is the observation error, which is not depended on the control input $u$. Recall that $e^{T} P(T) e \leq 1$ is the best possible estimate of the observation error. In this case, any control $u$, which guarantees

$$
\hat{\sigma}(T)=0
$$

is the solution of the optimization problem (4), where

$$
\hat{\sigma}:=f^{T} \hat{x}(T) .
$$

\section{A. The conventional sliding mode feedback}

Following the classical methodology of the sliding mode control design [22], [7] let us define

$$
u(t)=u_{e q}+u_{d}
$$

where

$$
u_{e q}(t)=-(f b)^{-1} f^{T} A x(t)
$$

is the so-called equivalent control part and

$$
u_{d}(t)=-(f b)^{-1} K(t) \operatorname{sign}[\sigma(t)],
$$

is the discontinuous (relay) term with sufficiently large positive function $K$.

Since the only observed state is admissible then the following laws must be applied

$$
\begin{aligned}
u_{e q}(t) & =-(f b)^{-1} f^{T} A \hat{x}(t), \\
u_{d}(t) & =-(f b)^{-1} K(t) \operatorname{sign}[\hat{\sigma}(t)] .
\end{aligned}
$$

Multiplying both sides of the system (8) by $f^{T}$ we obtain the following equation

$$
\frac{d \hat{\sigma}}{d t}=f^{T} A \hat{x}+f^{T} P(t) C^{T} R(y(t)-C \hat{x})+f^{T} b u(t),
$$

which defines the dynamic of sliding variable $\hat{\sigma}$ for the observer state space. Substituting the representation (13) for the control law we derive

$$
\frac{d \hat{\sigma}}{d t}=f^{T} P(t) C^{T} R(y(t)-C \hat{x})-K(t) \operatorname{sign}[\hat{\sigma}(t)]
$$

Taking into account $\hat{x}(0)=0$ we derive that for any

$$
K(t)>\left|f^{T} P(t) C^{T} R(y(t)-C \hat{x})\right|
$$

the control (13) guarantees achievement of the aim (11).

Therefore, the convectional sliding mode control is the solution of the optimization problem (4) if the design technique is based on minimax observer application. The formula (16) represents the rule for selection of the relay feedback gain.

Evidently, the simple modification of the control laws (14)

$$
\begin{aligned}
u_{e q}(t) & =-(f b)^{-1} f^{T}\left[A x(t)+P(t) C^{T} R(y(t)-C \hat{x})\right], \\
u_{d}(t) & =-(f b)^{-1} K(t) \operatorname{sign}[\hat{\sigma}(t)],
\end{aligned}
$$

also will gives us one more solution of the optimization (4) for any positive $K(t)$. Since the amplitude of "chattering" of the closed-loop system with the sliding mode control is usually proportional to the relay gain, then this modified sliding mode control is expected to be more reliable for practice. 


\section{B. The optimal continuous control}

Evidently, the continuous control can also be designed in order to guarantee the condition (11). For instance, the continuous feedback law

$$
u_{c}(t)=-\left(f^{T} b\right)^{-1} f^{T}\left[A \hat{x}(t)+P(t) C^{T} R(y(t)-C \hat{x}(t)]\right.
$$

is also optimal for the problem (4). Indeed, this feedback provides

$$
f^{T} \frac{d \hat{x}}{d t}=0
$$

So, taking into account $\hat{x}(0)=0$ we obtain $\hat{x}(T)=0$.

A class of optimal feedbacks can be generated by the following formula

$$
u_{o p t}(t)=u_{c}(t)-\left(f^{T} b\right)^{-1} \mathrm{~h}(\hat{\sigma}(t)),
$$

where $\mathrm{h}: \mathbb{R} \rightarrow \mathbb{R}, \mathrm{h}(0)=0$ is an odd function.

Introducing the function $h$ to the feedback law may be important for practical implementation of the optimal control law in order to provide an additional robustness of the closedloop system with respect to parametric disturbances and small non-linear effects.

For example, the nonlinear function providing finite-time stabilizing control (see, for example, [3], [14]) has the form

$$
\mathrm{h}_{F T}(z)=p|z|^{\alpha} \operatorname{sign}[z]
$$

with $\alpha \in(0,1), p>0$.

The fixed-time control algorithm (see [15], [12]) can be design using the function

$$
\mathrm{h}_{F x T}(z)=\left(p_{1}|z|^{\alpha}+p_{2}|z|^{\beta}\right) \operatorname{sign}[z]
$$

where $\alpha \in(0,1), \beta>1, p_{1}>0$ and $p_{2}>0$.

In the partial cases, when $\alpha \rightarrow 1$ and $\beta \rightarrow 1$ the presented control laws become linear feedbacks.

\section{NUMERICAL SimUlations}

Consider the linearized model of the inverted pendulum of the form

$$
A=\left(\begin{array}{ll}
0 & 1 \\
1 & 0
\end{array}\right), \quad b=\left(\begin{array}{l}
0 \\
1
\end{array}\right), \quad C=\left(\begin{array}{ll}
1 & 0
\end{array}\right)
$$

and select the desired sliding surface $f^{T} x=0$ with

$$
f=\left(\begin{array}{l}
1 \\
1
\end{array}\right)
$$

Assume, in addition, that the control input is bounded as follows

$$
|u(t)| \leq 10
$$

A. Comparison of linear ans sliding mode feedbacks based on minimax observer

The restrictions to energy measurement noises, exogenous disturbances and uncertainty of initial conditions is represented by the inequality (3) with the following parameters

$$
P_{0}=\frac{3 \pi^{2}}{4}\left(\begin{array}{ll}
1 & 0 \\
0 & 1
\end{array}\right), \quad Q=R=40 / 3, \quad T=10 .
$$

We compare the linear continuous feedback

$$
u_{\text {lin }}(t)=u_{c}(t)-\left(f^{T} b\right)^{-1} \hat{\sigma}(t)
$$

with the sliding mode control of the form (17) with $K=1$.

The initial condition selected for simulation is

$$
x_{0}=\left(\begin{array}{ll}
0 & \frac{\pi}{2}
\end{array}\right)^{T} .
$$

The deterministic noise and disturbance functions are defined by

$$
w(t)=0.05 \operatorname{sign}[\sin (2 t)] \quad \text { and } \quad g(t)=0.05 \operatorname{sign}[\cos (t)] .
$$

The numerical simulations has been made using explicit Euler method with fixed step size $h=0.01$.

The figures 1-3 presents the simulation results. Since the obtained results are almost identical for continuous and sliding mode control application we present them only for sliding mode case. As usual sliding mode just produces some small chattering behavior for the .
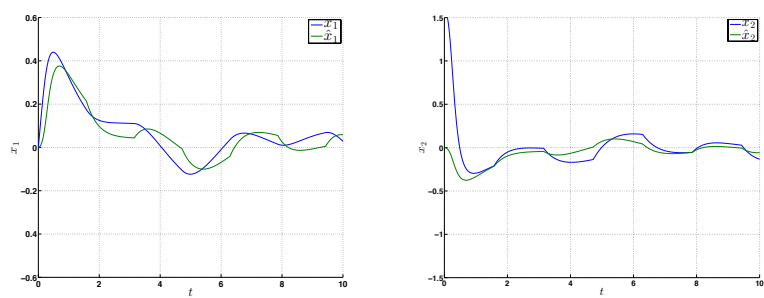

Fig. 1. The real and observed system states .
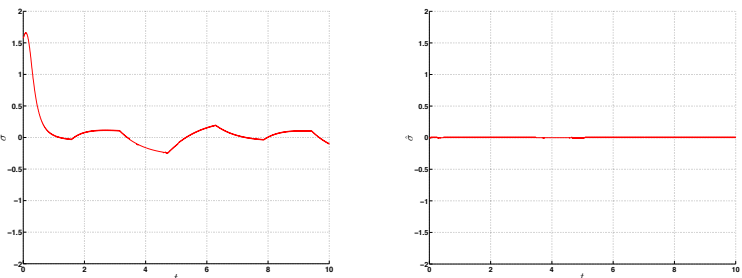

Fig. 2. The real(left) and observed(right) sliding variable .

B. Comparison of minimax observer and the second order sliding mode-based observer

The minimax observer is the best for the class of observers generated by linear functionals. It is important to compare the minimax observer with some nonlinear one. It is worth to stress that observation of the considered linear system can 

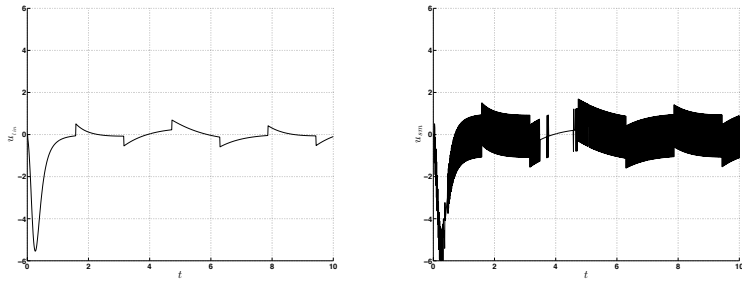

Fig. 3. The linear(left) and sliding mode(right) laws.

be done by means of the so-called second order sliding mode (2-sm) observer(differentiator) [11], [5] defined by

$$
\left\{\begin{array}{l}
\dot{z}_{1}(t)=-\gamma_{1} \sqrt{\left|z_{1}(t)-y(t)\right|} \operatorname{sign}\left[z_{1}(t)-y(t)\right]+z_{2}(t), \\
\dot{z}_{2}(t)=-\gamma_{2} \operatorname{sign}\left[z_{1}(t)-y(t)\right]+y(t)+u(t),
\end{array}\right.
$$

where $\gamma_{1}, \gamma_{2}$ are positive numbers.

The equation for the observation error dynamic has the form

$$
\left\{\begin{array}{l}
\dot{e}_{1}(t)=-\gamma_{1} \sqrt{\left|e_{1}(t)-w(t)\right|} \operatorname{sign}\left[e_{1}(t)-w(t)\right]+e_{2}(t), \\
\dot{e}_{2}(t)=-\gamma_{2} \operatorname{sign}\left[e_{1}(t)-w(t)\right]+g(t)
\end{array}\right.
$$

where $e_{1}=z_{1}-x_{1}, e_{2}=z_{2}-x_{2}$.

Following the paper [16] we select the observer parameters as follows

$$
\gamma_{1}=5.7446 \quad \text { and } \quad \gamma_{2}=6.1250
$$

For the comparison we use the fixed-time control of the form

$u_{F x T}=-\left(f^{T} b\right)^{-1}\left(f^{T} A z+\left(\left|f^{T} z\right|^{\frac{1}{2}}+\left|f^{T} z\right|^{\frac{3}{2}}\right) \operatorname{sign}\left[f^{T} z\right]\right)$,

where $z=\left(z_{1}, z_{2}\right)^{T}$.

Figure 4 depicts comparison results of the optimal linear feedback (22) and the fixed-time control (24). The simulation has been made for the same initial conditions, noises and disturbances using explicit Euler method with the step size $h=0.001$.
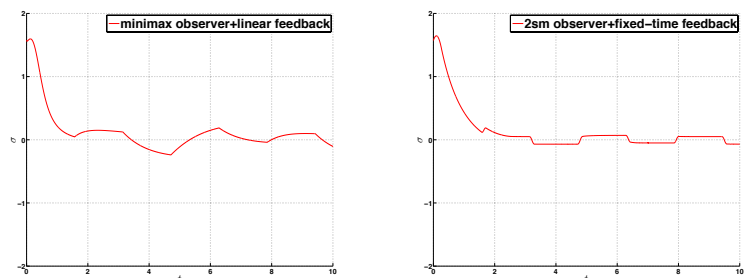

Fig. 4. Evolution of the sliding variable (deterministic noise).

Figure 5 shows the comparison results for random measurement noise, which was generated as a sequence of pseudorandom values drawn from the uniform distribution on the open interval $(-0.05,0.05)$.

Both experiments demonstrate better dynamic for nonlinear control scheme.
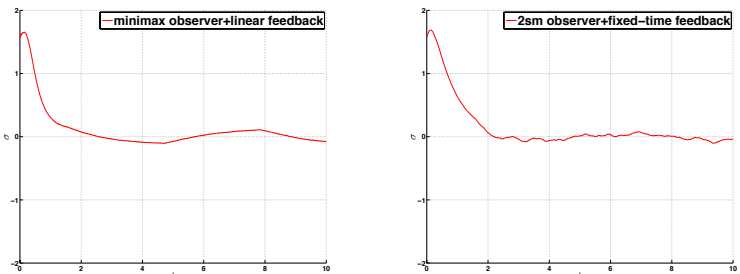

Fig. 5. Evolution of the sliding variable (random noise).

\section{CONCLUSION}

The problem of the optimal reaching (as close as possible) of the selected sliding surface for the linear time-invariant disturbed system with the noised measurements of the output is studied using minimax observation approach.

The following important facts were discovered:

- The conventional sliding mode control is an optimal solution to the considered problem.

- The optimal control law is not unique. The class of continuous optimal feedbacks is presented.

The performance of the designed optimal control laws is confirmed by numerical simulations.

In order to compare the minimax (linear) approach to observer design with nonlinear observation scheme based on high order sliding mode principles, the fixed-time (nonlinear) stabilizing feedback was designed using 2 -sm observer. The numerical simulations demonstrated better performance of the nonlinear scheme. This motivates us for future research in the area optimal nonlinear observation using sliding mode methodology. On the other hand, it would be interesting to design efficient linear feed-back strategies for Riccati equations in order to control the radius of the uncertainty ellipsoid.

\section{APPENDIX}

Sketch of the proof of Proposition 1.

The proof is based on the Kalman duality principle (see, [8]) which allows to prove that the minimax estimate of $l^{T} x(T)$ may be computed by introducing a linear quadratic dual control problem represented by a quadratic cost functional

$$
\begin{aligned}
I(U(\cdot)) & =z^{T}(0) P_{0} z(0) \\
& +\int_{0}^{T} U^{T}(\tau) R^{-1} U(\tau)+z^{T}(\tau) Q^{-1} z(\tau) d \tau
\end{aligned}
$$

over a linear adjoint differential equation:

$$
\frac{d z(t)}{d t}=-A^{T} z(t)+C^{T} U(t), \quad z(T)=l .
$$

In fact, the cost $I(U)$ represents the worst-case estimation error $\sigma(U, l, T)$ for an estimate $U$. It is quite natural then to minimize $I$ in order to find $\widehat{U}$ which has the minimal worstcase estimation error, so the minimax error $\sigma(\widehat{U}, l, T)$.

Now, using classical results in linear quadratic optimal control one finds the representation for $\widehat{U}$ in feed-back form: 
$\widehat{U}=R C P(t) z(t)$. The latter representation is then used, in turn, in order to prove, by integrating by parts, that:

$$
\widehat{\mathcal{U}}_{l}(y):=\int_{0}^{T} \widehat{U}^{T}(\tau) y(\tau) d \tau=l^{T} \hat{x}_{g}(T)
$$

and $\hat{\sigma}(l, T)=\left(l^{T} P(T) l\right)^{\frac{1}{2}}$, where $y_{g}$ is defined by (6).

Generally speaking, the construction of minimax observer (1)-(7) partially repeats the design of Kalman-Bucy filter (see, [9]), where instead of stochastic noises (stochastic measure) we consider the deterministic ones (Lebesgue measure).

\section{REFERENCES}

[1] J. M. Andrade-Da Silva, C. Edwards, and S. K. Spurgeon. Slidingmode output-feedback control based on lmis for plants with mismatched uncertainties. IEEE Transaction on Industrial Electronics, 56(9):3675-3683, 2009.

[2] V. Azhmyakov, A. Polyakov, and A. Poznyak. Consistent approximations and variational description of some classes of sliding mode control processes. Journal of The Franklin Institute, in press:http://dx.doi.org/10.1016/j.jfranklin.2013.01.011, 2014.

[3] S.P. Bhat and D.S. Bernstein. Finite-time stability of continuous autonomous systems. SIAM Journal of Control and Optimization, 38(3):751-766, 2000.

[4] F.L. Chernousko. State Estimation for Dynamic Systems. Boca Raton, FL: CRC, 1994.

[5] J. Davila, L. Fridman, and Levant A. Second-order sliding-mode observer for mechanical systems. IEEE Transactions on Automatic Control, 50(11):1785-1789, 2005.

[6] C. Edwards, A. Akoachere, and S. K. Spurgeon. Sliding-mode output feedback controller design using linear matrix inequalities. IEEE Transactions on Automatic Control, 46(1):115-119, 2001.

[7] C. Edwards and S. Spurgeon. Sliding Mode Control: Theory And Applications. CRC Press., 1998.

[8] R. E. Kalman. On the general theory of control systems. In Proccedings of the First Congress of Automatic Control. Moscow, USSR, 1960.

[9] R. E. Kalman and R.S. Bucy. New results in linear filtering and prediction theory. Journal of Basic Engineering, 83:95-108, 1961.

[10] A. Kurzhanski and I. Valyi. Ellipsoidal calculus for estimation and control. Birkhauser Boston Inc., Boston, MA, 1997.

[11] A. Levant. Robust exact differentiation via sliding mode technique. Automatica, 34 (3):379-384, 1998.

[12] A. Levant. On fixed and finite time stability in sliding mode control. In IEEE 52nd Conference on Decision and Control, pages 4260-4265, 2013.

[13] M. Milanese and R. Tempo. Optimal algorithms theory for robust estimation and prediction. IEEE Transactions on Automatic Control, 30(8):730-738, 1985.

[14] E. Moulay and W. Perruquetti. Finite time stability and stabilization of a class of continuous systems. Journal of Mathematical Analysis and Application, 323(2):1430-1443, 2006.

[15] A. Polyakov. Nonlinear feedback design for fixed-time stabilization of linear control systems. IEEE Transactions on Automatic Control, 57(8):2106-2110, 2012.

[16] A. Polyakov and A. Poznyak. Reaching time estimation for "supertwisting" second order sliding mode controller via Lyapunov function designing. IEEE Transactions on Automatic Control, 54(8):1951$1955,2009$.

[17] A. Polyakov and A. Poznyak. Invariant ellipsoid method for minimization of unmatched disturbance effects in sliding mode control. Automatica, 47(7):1450-1454, 2011.

[18] A.S. Poznyak. Variable Structure Systems: from Principles to Implementation, volume 66 of IEE Control Series, chapter Deterministic output noise effects in sliding mode observation, pages 45-78. The IET, London, UK, 2004.

[19] A.S. Poznyak. Advanced Mathematical Tools for Automatic Control Engineers: Deterministic Techniques. Elsevier, 2008.

[20] Y. Shtessel, C. Edwards, L. Fridman, and A. Levant. Sliding Mode Control and Observation. Birkhauser, 2014.
[21] V.I. Utkin. Variable structure systems with sliding modes. IEEE Transactions on Automatic Control, 22(2):212 - 222, 1977.

[22] V.I. Utkin, J. Guldner, and J. Shi. Sliding Mode Control in ElectroMechanical Systems. CRC Press., 2009.

[23] J. Yang, S. Li, and X. Yu. Sliding-mode control for systems with mismatched uncertainties via a disturbance observer. IEEE Transactions on Automatic Control, 60(1):160-169, 2013.

[24] S. Zhuk and M. Petreczky. Infinite horizon control and minimax observer design for linear daes. In Proc. 52nd IEEE Conference on Decision and Control, 2013. 